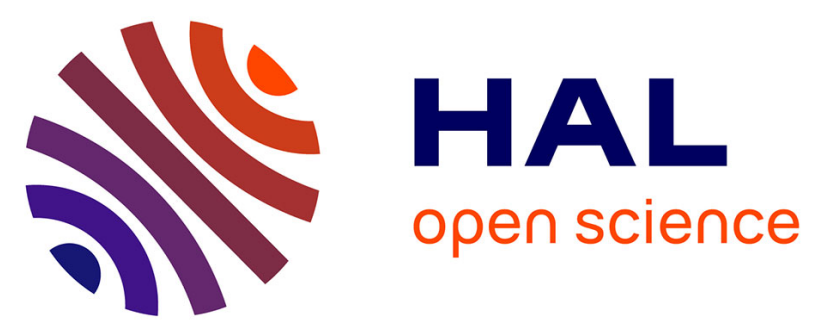

\title{
gene therapy enhances the therapeutic efficacy of doxorubicin to combat growth of MCF-7 breast cancer cells
}

Jose Prados, Consolación Melguizo, Ana Rosa Rama, Rául Ortiz, Ana Segura, Houria Boulaiz, Celia Vélez, Octavio Caba, Juan Luís Ramos, Antonia Aránega

\section{To cite this version:}

Jose Prados, Consolación Melguizo, Ana Rosa Rama, Rául Ortiz, Ana Segura, et al.. gene therapy enhances the therapeutic efficacy of doxorubicin to combat growth of MCF-7 breast cancer cells. Cancer Chemotherapy and Pharmacology, 2009, 66 (1), pp.69-78. 10.1007/s00280-009-1135-1 . hal00568271

\section{HAL Id: hal-00568271 \\ https://hal.science/hal-00568271}

Submitted on 23 Feb 2011

HAL is a multi-disciplinary open access archive for the deposit and dissemination of scientific research documents, whether they are published or not. The documents may come from teaching and research institutions in France or abroad, or from public or private research centers.
L'archive ouverte pluridisciplinaire HAL, est destinée au dépôt et à la diffusion de documents scientifiques de niveau recherche, publiés ou non, émanant des établissements d'enseignement et de recherche français ou étrangers, des laboratoires publics ou privés. 
Gef gene therapy enhances the therapeutic efficacy of doxorubicin to combat growth of MCF-7 breast cancer cells

Jose Prados*, Consolación Melguizo*, Ana Rosa Rama, Rául Ortiz, Ana Segura, Houria Boulaiz, Celia Vélez, Octavio Caba, Juan Luís Ramos, Antonia Aránega

Jose Prados, Consolación Melguizo, Ana Rosa Rama, Rául Ortiz, Houria Boulaiz, Celia Vélez, Antonia Aránega

Institute of Biopathology and Regenerative Medicine (IBIMER), Department of Human Anatomy and Embryology, School of Medicine, University of Granada, Granada, Spain.

Octavio Caba

Department of Health Sciences, University of Jaén, Spain.

Ana Segura, Juan Luis Ramos

CSIC-Estación Experimental del Zaidín, Department of Environmental Protection, Granada E18008, Spain.

* These authors contributed equally to this work

Corresponding author and reprint requests: Dr. J. Prados, Instituto de Biopatología y Medicina Regenerativa (IBIMER), Dept. Anatomía y Embriología, Facultad de Medicina, Universidad de Granada, E-18071 GRANADA, Spain

Tel. :+34-958-243534

Fax:+34-958-246296 E-mail jcprados@ ugr.es

Keywords Gene therapy, gef gene, Cytotoxic therapy, Breast cancer, Multicellular tumour spheroid, Doxorubicin 


\begin{abstract}
Purpose The potential use of combined therapy is under intensive study including the association between classical cytotoxic and genes encoding toxic proteins which enhanced the antitumour activity. The main aim of this work was to evaluate whether the gef gene, a suicide gene which has a demonstrated antiproliferative activity in tumor cells, improved the antitumour effect of chemotherapeutic drugs used as first line treatment in the management of advanced breast cancer.
\end{abstract}

Methods MCF-7 human breast cancer cells were transfected with gef gene using pcDNA3.1TOPO expression vector. To determine the effect of the combined therapy, MCF-7 transfected and non transfected cells were exposed to paclitaxel, docetaxel and doxorubicin at different concentrations. The growth-inhibitory effect of gef gene and/or drugs was assessed by MTT assay. Apoptosis modulation was determined by flow cytometric analysis, DNA fragmentation and morphological analysis. Multicellular tumour spheroids (MTS) from MCF-7 cells were used to confirmed effectiveness of combined therapy (gef gene and drug).

Results Our results demonstrate that combined therapy gef gene/drugs (paclitaxel, docetaxel or doxurubicin) caused a decrease in cell viability. However, only the gef-doxorubicin $(10 \mu \mathrm{M})$ combination induced a greater enhancement in the antitumour activity in MCF-7 cells. Most importantly, this combined strategy resulted in a significant synergistic effect, thus allowing lower doses of the drug to be used to achieve the same therapeutic effect. These results were confirmed using MTS in which volume decrease with combined therapy was greater than obtained using the gene therapy or chemotherapy alone, or the sum of both therapies.

Conclusions The cytotoxic effect of gef gene in breast cancer cells enhances the chemotherapeutic effect of doxorubicin. This therapeutic approach has the potential to overcome some of the major limitations of conventional chemotherapy, and may therefore constitute a promising strategy for future applications in breast cancer therapy. 


\section{Introduction}

Metastatic breast cancer (MBC) remains an incurable illness. The primary goal of its management is palliative and to improve the quality of life, prolong disease-free survival and possibly overall survival [1]. Chemotherapy clearly provides tumour shrinkage and substantial clinical benefit in advanced breast cancer [2], and is accepted as standard treatment for hormone-resistant and rapidly progressive disease. Several chemotherapeutic options have been shown to be effective as first- or second-line therapy in the management of MBC with taxanes, such as paclitaxel (Pacl) and docetaxel (Doc) and anthracyclines, such as doxorubicin (Dox), being the most active drugs, although with substantial side effects [3]. An important area of cancer research seeks to improve the effectiveness of these cytotoxic agents in breast cancer and to reduce their side effects [4].

Gene therapy has been proposed as a strategy to enhance the antitumour effect of drugs in the treatment of refractory or advance cancers. The majority of these studies have assessed classical gene therapy systems such as herpes simplex virus thymidine kinase (HSVtk) [5, 6] and cytosine deaminase $(C D)$ genes $[7,8]$ from $S$. Coli. However, their results had two important limitations, the need to convert a non-toxic prodrug into a toxic metabolite and the limited bioavailability of the active drug metabolite $[9,10]$. A new strategy that may avoid this problem is the use of therapeutic genes that directly encode cytotoxic proteins. In contrast to classic suicide genes, which act by disrupting DNA synthesis and therefore target only rapidly dividing cells, these new toxins may act by killing both quiescent and rapidly dividing tumour cells and may be effective for aggressively growing tumours as well as for those that grow more slowly. The most recent experiences with genes from bacteria [11], bacteriophages [12], viruses [13] or plants [14] expressing toxins showed significant antitumour activity. Their potential use in combined chemotherapy and/or radiotherapy is now being assessed. 
Our group has recently developed a new cancer gene therapy strategy using a toxic gene from the chromosome of $E$. coli $(g e f)$ which does not need a prodrug to be effective in tumour cells. The gef gene, a member of a gene family with homologous cell-killing functions, encodes a membrane protein of 50 amino acids that is anchored in the cytoplasmic membrane by the $\mathrm{N}$-terminal portion. Activation of this protein induces arrest of cellular respiration and cell death [15]. In human tumour cells, gef gene induces cell cycle arrest and apoptosis [16, 17], which may be a complementary strategy for classic cancer treatments, since it has been demonstrated that apoptosis deficiency is a critical factor for treatment failure in advanced cancer [18]. In this context, gef gene may improve current chemotherapy options in cancer, including MBC, and could be of use as an adjuvant therapy to increase the effectiveness of conventional tumour treatment.

The aim of this study is to investigate whether gef gene enhances the antitumour effect of drugs used as a first line therapy in MBC. Our results suggest gef gene could be a new tool in the design of gene therapy strategies against MBC, by increasing the effectiveness of classic drugs. The chemotherapeutic potential of gef gene/Dox combination in human breast cancer treatment merits a thorough evaluation.

\section{Methods}

Cell culture

The MCF-7 breast carcinoma cell line (European Collection of Cell Culture) was grown with DEMEM (Sigma Chemical Co., St. Louis, MO), supplemented with $10 \%$ heat-inactivated foetal bovine serum (FBS), $40 \mathrm{mg} / \mathrm{l}$ gentamicin and $500 \mathrm{mg} / \mathrm{l}$ ampicillin (Antibióticos S.A, Madrid, Spain). Cells were maintained in monolayer culture at $37^{\circ} \mathrm{C}$ in an atmosphere containing $5 \% \mathrm{CO}_{2}$. 
Vector construction

The gef gene was kindly provided by Dr J.L. Ramos from the Zaidín Experimental Station, CSIC, Granada, Spain. After its amplification using specific primers (sense 5'ATGAAGCAGCATAAGGCGATG-3' and antisense 5'-TTACTCGGATTCGTAAGCCGTC3'), gef gene was subcloned into the pcDNA3.1-TOPO vector following manufacturer's instructions (Invitrogen, Barcelona, Spain). The resulting plasmid pcDNA3.1/gef was confirmed by sequence analysis using the T7 primer 5'-TAATACGACTCACTATAGGG-3'. Plasmid DNA was amplified in E. coli DH5 $\alpha$ and purified by large-scale plasmid preparation using columns (Qiagen, Barcelona, Spain). To optimise transfection conditions, the pcDNA3.1-TOPO/lacZ (encoding $\beta$-galactosidase) and X-GAL staining was used. To detect gef protein, a fusion protein (gef-GFP) was generated using the plasmid pcDNA3.1/GFP (provided by Dr. G. Ortiz). The gef cDNA was obtained by PCR, modifying the reverse primer to eliminate the stop codon, and ligated into pcDNA3.1/GFP vector following the manufacturer's protocol (Invitrogen). The resulting plasmid pcDNA3.1/GFP-gef was confirmed and amplified as described above.

Transfection

One day before transfection, confluent cells were seeded into 6 -well plates $\left(2 \times 10^{5}\right.$ cells per well). Briefly, a transfection mixture was prepared by adding $94 \mu \mathrm{l}$ of the serum-free medium and $6 \mu$ FuGENE-6 reagent (Roche Diagnostic, Barcelona, Spain). After 5 min incubation at room temperature, $2 \mu \mathrm{g}$ of plasmid DNA were added (ratio 1:3). MCF-7 cells, yielding approximately $70 \%$ confluence, were transfected pcDNA3.1/gef. Cells were cultivated for $8 \mathrm{~h}$ at $37^{\circ} \mathrm{C}$ and the medium containing transfection mixture was then replaced with the growth medium. A pcDNA3.1-TOPO plasmid in which the gef gene was absent was used as a negative 
control. The transfection of pcDNA3.1/GFP-gef to determine gef protein was realized as $\underline{\text { described above. }}$

Reverse Transcription-PCR (RT-PCR)

Upregulation of mRNA expression of gef cDNA was determined by RT-PCR. Total RNA was extracted from transfected and parental cells with the Rneasy Mini kit (Qiagen, Madrid, Spain), and cDNA was generated by means of the Promega reverse transcription system (Promega, Madrid, Spain) using total cellular RNA $(1 \mu \mathrm{g})$. PCR amplification of gef gene took place under the above-described conditions and was run on a $1.8 \%$ agarose gel and visualized by ethidium bromide staining. RNA integrity was assessed by amplification of $\beta$-actin mRNA. Images were scanned and analysed using the Quantity One Analysis Software (Bio-Rad Laboratories, Inc., Barcelona, Spain).

\section{Microscopic Analysis and Western Blotting}

gef protein expression was confirmed in MCF-7 pcDNA3.1/GFP-gef transfected cells with excitation at $488 \mathrm{~nm}$. Fluorescent microscopy analys is was carried out with a Nikon Eclipse Ti (Nikon Instruments Inc. NY, USA). Protein extracts $(30 \mu \mathrm{g})$ from parental and transfected cells were used for SDS-PAGE in a Mini Protean II cell (Bio-Rad, Hercules, CA). The separated proteins were transferred to a nitrocellulose membrane (20 V at room temperature for $30 \mathrm{~min})$ and the blots treated with blocking solution ( $20 \mathrm{mM}$ Tris, $0.9 \mathrm{NaCl}, 10 \%$ non-fat milk) for $3 \mathrm{~h}$. GFP-gef fusion protein was detected with an Anti-GFP N-terminal antibody (Sigma, St. Louis, MO). 


\section{Proliferation Assay}

Parental and transfected MCF-7 cells were seeded in 48 well plates at a density of $5 \times 10^{3}$ cells per well. In order to determine the effect of the combined therapy (gef gene and drugs: Pacl, Doc and Dox) they were treated with gradient concentrations $(0.1,1$ and $10 \mu \mathrm{M})$ according to the recommended dose for patients [19]. After 24h, 48h and 72h, $20 \mu \mathrm{L}$ MTT (3-(4,5dimethylthiazol-2-yl)-2,5-diphenyltetrazolium bromide) solution $(5 \mathrm{mg} / \mathrm{ml})$ was added to each well and incubated at $37^{\circ} \mathrm{C}$ for $4 \mathrm{~h}$. Then $200 \mu \mathrm{l}$ of dimethylsulphoxide (DMSO) was added to each well after the medium was removed. Absorbances were then measured using a Titertek multiscan colorimeter (Flow, Irvine, CA) at 570 and $690 \mathrm{~nm}$. Linearity of the MTT assay with cell number was tested for each MCF-7 cell stock before each cell growth experiment.

Apoptosis analysis

Cells were washed twice with PBS and incubated in binding buffer containing annexin V-FITC $(25 \mu \mathrm{g} / \mathrm{ml})$ and PI $(25 \mu \mathrm{g} / \mathrm{ml})$ in the dark for $15 \mathrm{~min}$ at room temperature (Annexin V-FITC Apoptosis Detection Kit I, BD Pharmingen, San Diego, CA). Binding buffer (500 $\mu$ l) was then added and cells were immediately processed with a FACScan flow cytometer (Becton Dickinson, San Jose, CA). Microscopy analysis was carried out in a Leica DMI6000 confocal microscope with an Argon/Krypton laser (Heidelberg, Germany).

\section{DNA fragmentation assay}

After the treatments, MCF-7 cells were lysed in a buffer containing $20 \mathrm{mM}$ Tris $\mathrm{pH} 8.5,5 \mathrm{mM}$ EDTA, $10 \%$ sodium dodecyl sulphate (SDS), and $100 \mu \mathrm{g} / \mathrm{ml}$ proteinase $\mathrm{K}$ and incubated overnight at $37^{\circ} \mathrm{C}$. Lysates were cleared by centrifugation at $12000 \mathrm{~g}$ for $20 \mathrm{~min}$. DNA in the supernatant was extracted with an equal volume of neutral phenol:chloroform:isoamyl alcohol 
mixture (25:24:1), precipitated with $95 \%$ ethanol for $2 \mathrm{~h}$ at $-80^{\circ} \mathrm{C}$ and analysed by agarose gel electrophoresis.

\section{MTS initiation}

To generate multicellular tumour spheroids (MTS), exponentially growing monolayer MCF-7 cells were harvested by trypsinisation. Then $2 \times 10^{3}$ cells/well were grown in a 48 -well plate (BD) previously coated with $200 \mu 10.75 \%$ agarose type II in FBS-free medium and allowed to dry for $30 \mathrm{~min}$. Plates were incubated at $37^{\circ} \mathrm{C}$ in a $5 \% \mathrm{CO}_{2}$ atmosphere to promote aggregation and transferred into a rocker designed for three-dimensional agitation (70 cycles/min) as described previously [20]. A single MTS of approximately $100 \mu \mathrm{m}$ of diameter was obtained in each well. Growth of the spheroids was monitored and measured using an inverted phase contrast microscope to obtain a median relative volume (volume at day x/volume at day 0 ), which was calculated as previously described by Monazzam et al. [19]. When the MTS grow to a considerable size they also become asymmetric, thus the time for their evaluation was set for 7 days.

\section{Transfection in MCF-7 MTS}

MTS cells were transferred using a Pasteur pipette from the 48-well microplate to a 96-well plate (one MTS per well) coated with agarose and containing $100 \mu \mathrm{l}$ of medium. To perform the combined therapy experiments, the MTS were transfected with pcDNA3.1/gef in two different ways, on the first day of the experiment only (MTS-FD) and every two days during the experiment (days 1, 3 and 5) (MTS-ETD). MCF-7 spheroids transfected with empty vector were used as a control. The response to treatment was evaluated by measuring MTS volume as described above. 


\section{Combined therapy in MCF-7 MTS}

MTS in a 96-well plate microplate (one MTS per well) were analysed in four groups: nontreated MTS, drug-treated non-transfected MTS, drug-treated transfected MTS-FD and drugtreated transfected MTS-ETD. The drugs (Pacl, Doc and Dox) were used at the same doses described above. The response to each anticancer treatment was evaluated by measuring MTS volume during treatment.

\section{Statistical analysis}

SPSS 7.5 software (SPSS, Chicago, IL) was used for all statistical analyses. Results were compared by using Student's $t$-test. All data are expressed as means \pm SD. Differences were considered statistically significant at a $P$-value of $<0.05$.

\section{Results}

In vitro evaluation of $g e f$ gene expression

In vitro evaluation of gef gene expression was performed by RT-PCR. As shown in Fig. 1, an amplification fragment of $154 \mathrm{bp}$ was found in the MCF-7 cells transfected with pcDNA3.1/gef, indicating the effectiveness and ability of the construction to be used in the subsequent in vitro experiment. To demonstrate the integrity of the RNA preparations, PCR was performed using $\beta$-actin primers (Fig. 1). GFP-gef protein was detected in the cytoplasm after transfection of MCF-7 cells with pcDNA3.1/GFP-gef (Fig.1).

Effects of the combined therapy on MCF-7 cell proliferation

The main goal of this study was to evaluate the therapeutic potential resulting from the combination of two antitumour strategies, suicide gene therapy (gef gene) and conventional 
chemotherapy (Pacl, Doc and Dox), in breast cancer cells. For this purpose, MCF-7 cells were exposed to classic chemotherapy agents for breast cancer treatment in non transfected and transfected cells. As shown in Fig. 2, gef gene was able to induce a continuous decrease in the MCF-7 cell viability in the absence of drugs. After $72 \mathrm{~h}$ transfection a $31 \%$ decrease in cell viability was observed. Non-transfected cells treated with Dox, Pacl and Doc at different concentrations also showed a significant decrease in cell viability (Fig. 2). However, transfected MCF-7 cells in the presence of drugs showed a greater decrease in survival population compared to the results obtained with drugs or gef gene alone. The most interesting finding was obtained when Dox was used in transfected cells, as this combined therapy not only induced just the sum of drug and gef individual treatments but there was also a significant enhancement of the antitumour effect. As shown in Fig. 2A, the combined therapy gef/Dox (10 $\mu \mathrm{M})$ produced a 50, 83 and $96 \%$ decrease in MCF-7 cell viability at 24, 48 and $72 \mathrm{~h}$, respectively, when compared to control cells. Therefore, this combined therapy had $15 \%$ more antitumour effect at $24 \mathrm{~h}$ than the sum of both gef gene (12\%) and Dox $10 \mu \mathrm{M}(23 \%)$ treatments together (Fig. 2A). This enhancer phenomenon was also observed at $48 \mathrm{~h}$ (18\% more effect) and $72 \mathrm{~h}(10 \%$ more effect). Similar results were obtained with Dox at $1 \mu \mathrm{M}$ although the enhancer effect on cell viability was slightly less than the sum of the both treatments (Fig. 2A). Finally, Dox at $0.1 \mu \mathrm{M}$ only produced a mild enhancer effect (5-7\%) which was not significant. As shown in Figure 2B, Pacl at different concentrations in transfected cells induces a significant decrease in the cell viability that represents the sum of the effect of both treatments. A similar finding was obtained with Doc although at $1 \mu \mathrm{M}$ or $0.1 \mu \mathrm{M}$ this drug produced a slight greater effect in transfected MCF-7 cells (Fig. 2C). 
Apoptosis in MCF-7 cells by combined therapy

As only the gef/Dox combination produced a significant decrease in the viability of MCF-7, the apoptosis experiments were conducted with this combination. Individual treatment with both gef gene and Dox induced apoptosis in MCF-7 cells. As shown in Fig. 3A, a significant increase in apoptosis was found at $24 \mathrm{~h}, 48$ and $72 \mathrm{~h}$ after gef gene transfection (13, 28 and $38 \%$, respectively) in relation to non-transfected cells used as a control. This apoptosis induction was confirmed by a typical DNA ladder fragmentation pattern (Fig. 3B) and by confocal laser-scanning microscopy (Fig. 3C). On the other hand, MCF-7 cells treated with Dox showed a time- a concentration-dependent apoptosis increase (Fig. 4). However, transfected MCF-7 cells exposure to Dox showed a significant increase in apoptosis level in comparison to cells treated with gef or Dox alone (Fig. 4). The most significant findings were obtained with combined therapy $(g e f / D o x ~ 10 \mu \mathrm{M})$ which caused 61,88 and $95 \%$ apoptosis at 24, 48 and $72 \mathrm{~h}$, respectively. These results indicated that gef gene was able to enhance the apoptotic effect of Dox in MCF-7 more than the sum of apoptosis induced by gef and Dox individually. Similar findings were observed with lower doses of Dox although the apoptosis increase was lower $(1 \mu \mathrm{M})$ or not significant $(0.1 \mu \mathrm{M})$.

Growth modulation of $g e f$ gene transfected MTS-MCF-7 cells

Volume rate of MTS derived from MCF-7 cells was measured after $g$ ef transfection during 7 days in order to analyse the potential of this gene to decrease the growth of breast cancer cell spheroids. Volume rate of MTS transfected with empty vector was similar to that of nontransfected MTS (data not shown). In contrast, gef gene transfected MTS showed a significant and time-dependent decrease in growth rate in both MTS-FD (transfected only the first day) and MTS-ETD (transfected every two days) (see Methods). The greatest decrease in growth 
rate after treatment with gef gene was observed at 7 days (Fig. 5). At this time, a $17.2 \%$ and a $28.1 \%$ volume decrease was observed in MTS-FD and MTS-ETD, respectively.

Effects of the combined therapies on MCF-7-MTS growth

To confirm the therapeutic potential of combined gef/Dox therapy in breast cancer cells we evaluated the modulation of MTS growth. Doxorubicin at 10,1 and $0.1 \mu \mathrm{M}$ induced a clear growth rate inhibition in non-transfected MTS (Fig.6). However, the exposure of transfected MTS (both MTS-FD and MTD-ETD) to this drug induced a higher decrease in MTS volume than the treatment with gef or Dox alone. Moreover, the MTS growth inhibition was also greater that those obtained by the sum of both gef and drug treatments together, indicating a significant enhanced of antitumour effect. Treatment with Dox $(10 \mu \mathrm{M})$ in MTS-ETD produced greater growth inhibition than MTS-FD. In MTS-ETD, the largest decrease in volume (63\%) was obtained with Dox $10 \mu \mathrm{M}$ compared to the volume of MTS control. Gef gene also enhanced MTS growth inhibition when combined with Dox at 1 and $0.1 \mu \mathrm{M}$, although to a lesser extent (Fig. 6). Finally, gef/Pacl and gef/Doc combined treatment in MTS (MTS-FD and MTS-ETD) ind uced a volume reduction that represented the sum of the effect of gef and drug only, but did not enhance the antitumour effect (Fig. 6).

\section{Discussion}

The development of therapeutic strategies to improve overall survival in refractory advanced breast cancer remains a high priority. Drug combinations, including Pacl, Doc and Dox, are currently the most effective treatments in these patients, although with little impact in the prognosis and with substantial side effects [3]. In this study, we have demonstrated that the suicide gef gene combined with Dox, induced an adjuvant effect that enhances its antitumour 
effect in breast cancer cells. In addition, these results raise the possibility of a chemotherapy dose reduction when used in combination with gene therapy.

It has been demonstrated that the association of a taxane with an anthracycline improve treatment response of patients with MBC. Recent studies showed that patients receiving Doc after Dox had a 5-year disease-free survival rate higher than those receiving a combination of the two drugs at the same time [21]. Phase I and II studies with the Dox-Pacl sequence defined by preclinical studies have reported objective responses above $40 \%[22,23]$. These results are higher than those obtained with other drugs, including fluorouracil, epirubicin and cyclophosphamide (FEC) [24, 25]. However, due to the patient response failure, it remains a priority to increase drug effectiveness against breast cancer cells. In this context, gene therapy has been proposed as a strategy to enhance drug activity and reducing drug doses in advanced cancer [4].

Isolated gene therapy has not led to the successful treatment of breast cancer. Classic suicide genes such as CD and HSVtk, are able to decrease breast cancer cell proliferation, but the need to use a prodrug is its main limitation [26]. During the last few years, new genes that encode toxins with a direct antitumour action have been developed and are being studied as single treatments in tumour therapy and in combination with conventional tumour treatment strategies. These toxic genes have been successfully used in a wide variety of tumours such as pancreas cancer (diphtheria toxin gene) [11], lung cancer, glioma and hepatocellular carcinoma (streptolysin O gene) [27] and cervical cancer ( $\lambda$-holin gene) [28]. Some of these toxics genes, such as saporin, showed synergistic effects when used with a cytotoxic drug [14]. We have previously demonstrated that gef gene showed antitumour activity in breast cancer cells by inducing apoptosis [18]. Moreover, it has been demonstrated that gef gene is able to enhance the effect of drugs in lung cancer cells [20]. In fact, the increase of Pacl antitumour effect has been also demonstrated when combined with IL12 gene therapy in breast and in ovarian 
cancers [29]. Novel advances in the combined use of gene therapy and cytotoxic drugs have recently been reported in bladder cancer [7], pancreatic cancer [30], and colorectal cancer [31]. Gene therapy with a caspase activator (Smac/DIABLO] has been used in breast cancer to enhance apoptosis induced by paclitaxel, doxorubicin, etoposide and tamoxifen [32]. These findings suggest that the antiproliferative effect of some drugs may be enhanced by the use of the gene therapy.

Our results showed that a combination of gef with Pacl, Doc and Dox produced a decrease in MCF-7 cell viability higher than that obtained with individual treatments. However, the most significant finding was related to the gef gene capacity to enhance Dox antitumour effect. Doxorubicin at $10 \mu \mathrm{M}$ produced a decrease in cell viability of MCF-7 transfected cells greater $(15 \%$ more) than the sum of the individual effects of gef and Dox when used separately. In fact, genes such as P450 3A4 was able to induce antitumor activity potentiation of doxorubicin when was used in ovarian ( $\mathrm{CHO})$ and lung cancer cells (A-549) [33]. Interestingly, lower concentrations of Dox $(1 \mu \mathrm{M})$ induced a similar antiproliferative enhancer effect to that of higher concentrations of this drug, suggesting that gef/Dox combination may be useful for reducing the cytotoxic concentrations needed in breast cancer treatment. gef gene alone was able to inhibit cell proliferation via apoptosis, as evidenced by MTT assay, flow cytometry analysis, DNA fragmentation and apoptotic morphology analysis. Our results support previous studies which have demonstrated that Dox, as a classical DNAdamaging agent, induced apoptosis in a variety of tumor cell lines, including breast cancer cells [34]. The in vitro experiments designed to investigate whether gef gene could enhance the apoptosis of MCF-7 cells induced by Dox were positives, resulting in a decrease of MCF-7 cell viability in a similar way that pro-apoptotic mPNAS-4 gene enhanced the cisplatin activity in lung, colon and ovarian carcinoma cells [35]. In contrast, although combined gef/Pacl or gef/Doc therapy also produced a decrease in cell viability, this was the equal to the sum of the 
$\underline{\text { effects when gene and drug were used separately. Similar results were obtained for all Doc and }}$ $\underline{\text { Pacl concentrations tested. It remains unclear why gef does not enhance the antitumor activity }}$ of taxanes despite the fact that their apoptotic effects in breast cancer cells have been confirmed in numerous studies [36]. Future studies will therefore be necessary to determine the apoptosis induction pathway for $g e f$, which is probably related to potentiation of the effect of $\underline{\text { Dox but not Doc or Pacl. }}$

To confirm the effectiveness of gef/Dox combined therapy in breast cancer we used MTS from MCF-7 cells as an experimental system which mimics the real biological environment of a tumour, including limitations in drug or gene penetration [36] and distribution and feedback mechanisms in cell signalling [37]. In fact, MTS has been previously used to determine apoptosis and proliferation of MCF-7 cells after treatment with Dox, Doc and combined Dox/Doc therapy [38] and to study the efficacy of suicide gene therapy systems such us HS Vtk [39]. Volume analyses of the MTS showed that combined gef/drug therapy caused significant growth inhibition in comparison with MTS control. As in cell cultures, the most significant results were obtained with the use of Dox $(10 \mu \mathrm{M})$ after gef gene transfection. This combined therapy caused a volume reduction greater than the sum of individual gef and Dox treatments. These results confirmed a significant enhancement in MTS growth inhibition. Experiments using MTS with a continuous transfection (MTS-ETD) demonstrated that high pressure sensitive $g$ ef gene expression increased the efficacy of the combined therapy by reducing the MTS volume more than using transfection only (MTS-FD). Moreover, as in culture cells, Dox at $1 \mu \mathrm{M}$ after gef gene transfection also inhibited MTS growth more than the exclusive use of higher doses of Dox, supporting the hypothesis that the use of gef could reduce drug concentrations while obtaining the same antiproliferative result. However, as is the case with most of the toxic genes used in gene therapy, it will be necessary to create tumor specificity $\underline{\text { using enhancer/promoter genes, new vectors and direct in vivo administration (intratumoral }}$ 
injection). Future studies with gef gene integrated into an optimised vector with targeted cellular promoters, similar to that described by Brandtner et al. [40] to $\lambda$-holin toxic gene, will be necessary to improve the effectiveness of this combined therapy in breast cancer.

In summary, we have reported the ability of the gef gene to enhance the antiproliferative effect of Dox in MCF-7 cells. In addition, combined gef/Dox therapy enhanced cell growth inhibition of MTS derived from these breast cancer cells. The successful use of this experimental combined therapy in enhancing the anticancer effect suggests a potential application in patients with advanced breast cancer.

\section{Acknowledgments}

We thank Dr R. Luque of the Oncology Service, Virgen de las Nieves Hospital, Granada, for providing the chemotherapy treatment and Dr. G. Ortiz Ferrón for excellent technical assistance. This study was supported by the Fondo Investigaciones Sanitarias Seguridad Social (FIS; project no. PI041372) and by Granada University (project no. UGR-30 B364 1101/2007) and MEC (FPU predoctoral fellowship).

\section{Conflicts of inte rest statement}

No potential conflicts of interest were disclosed. 


\section{References}

1. Brenner H, Gondos A, Arndt V (2007) Recent major progress in long-term cancer patient survival disclosed by modeled period analysis. J Clin Oncol 25:3274-3280.

2. Moulder S, Hortobagyi GN (2008) Advances in the treatment of breast cancer. Clin Pharmacol Ther 83:26-36.

3. Coley HM (2008) Mechanisms and strategies to overcome chemotherapy resistance in metastatic breast cancer. Cancer Treat Rev 34:378-390.

4. Tang SC (2009) Strategies to decrease taxanes toxicities in the adjuvant treatment of early breast cancer. Cancer Invest 27:206-214.

5. Freund CT, Tong XW, Rowley D, Engehausen D, Frolov A, Kieback DG, Lerner SP (2003) Combination of adenovirus-mediated thymidine kinase gene therapy with cytotoxic chemotherapy in bladder cancer in vitro. Urol Oncol $21: 197-205$.

6. Mavria G, Harrington KJ, Marshall CJ, Porter CD (2005) In vivo efficacy of HSV-TK transcriptionally targeted to the tumour vasculature is augmented by combination with cytotoxic chemotherapy. J Gene Med 7:263-275.

7. Shieh GS, Shiau AL, Yo YT, Lin PR, Chang CC, Tzai TS, Wu CL (2006) Low-dose etoposide enhances telomerase-dependent adenovirus-mediated cytosine deaminase gene therapy through augmentation of adenoviral infection and transgene expression in a syngeneic bladder tumor model. Cancer Res 66:9957-9966.

8. Gopinath P, Ghosh SS (2008) Apoptotic induction with bifunctional E.coli cytosine deaminase-uracil phosphoribosyltransferase mediated suicide gene therapy is synergized by curcumin treatment in vitro. Mol Biotechnol 39:39-48.

9. Dachs GU, Tupper J, Tozer GM (2005) From bench to bedside for gene-directed enzyme prodrug therapy of cancer. Anticancer Drugs 16:349-359. 
10. Altaner C (2008) Prodrug cancer gene therapy. Cancer Lett 270:191-201.

11. Showalter SL, Huang YH, Witkiewicz A et al (2008) Nanoparticulate delivery of diphtheria toxin DNA effectively kills Mesothelin expressing pancreatic cancer cells. Cancer Biol Ther 7:1584-1590.

12. Agu CA, Klein R, Lengler J et al (2007) Bacterio-phage-encoded toxins: the lambda holin protein causes caspase-independent non-apoptotic cell death of eukaryotic cells. Cell Microbiol 9:1753-1765.

13. Zhao JM, Wen ZJ, Li Q et al (2008) A promising cancer gene therapy agent based on the matrix protein of vesicular stomatitis virus. FASEB J 22:4272-4280

14. Geden SE, Gardner RA, Fabbrini MS, Ohashi M, Phanstiel IO, Teter K (2007) Lipopolyamine treatment increases the efficacy of intoxication with saporin and an anticancer saporin conjugate. FEBS J 274:4825-4836.

15. Poulsen TT, Pedersen N, Poulsen HS (2005) Replacement and suicide gene therapy for targeted treatment of lung cancer. Clin Lung Cancer 6:227-236.

16. Boulaiz H, Prados J, Marchal JA et al (2003) Transfection of MS-36 melanoma cells with gef gene inhibits proliferation and induces modulation of the cell cycle. Cancer Sci 94:564568.

17. Boulaiz H, Prados J, Melguizo C et al (2003) Inhibition of growth and induction of apoptosis in human breast cancer by transfection of gef gene. Br J Cancer 89 :192-198.

18. Simstein R, Burow M, Parker A, Weldon C, Beckman B (2003) Apoptosis, chemoresistance, and breast cancer: insights from the MCF-7 cell model system. Exp Biol Med (Maywood) 228:995-1003.

19. Monazzam A, Josephsson R, Blomqvist C, Carls son J, Langström B, Bergström M (2007) Application of the multicellular tumour spheroid model to screen PET tracers for analysis of early response of chemotherapy in breast cancer. Breast Cancer Res 9:R45. 
20. Prados J, Melguizo C, Rama A et al (2008) Combined therapy using suicide gef gene and paclitaxel enhances growth inhibition of multicellular tumour spheroids of A-549 human lung cancer cells. Int J Oncol 33:121-127.

21. Francis P, Crown J, Di Leo A et al (2008) BIG 02-98 Collaborative Group. Adjuvant chemotherapy with sequential or concurrent anthracycline and docetaxel: Breast International Group 02-98 randomized trial. J Natl Cancer Inst 100:121-133.

22. Ibrahim T, Fabbri M, Frassineti GL, Zoli W, Monti M, Ricotti L, Amadori D (2003) Doxorubicin, paclitaxel and gemcitabine: a Phase I study of a new sequential treatment in stage III B - IV breast cancer. J Chemother $15: 488-494$.

23. Zielinski CC (2003) Gemcitabine, anthracycline, and taxane combinations for advanced breast cancer. Oncology (Huntingt) 17:36-40.

24. Burdette-Radoux S, Wood ME, Olin JJ, Laughlin RS, Crocker AM, Ashikaga T, Muss HB (2007) Phase I/II trial of adjuvant dose-dense docetaxel/epirubicin/cyclophosphamide (TEC) in stage II and III breast cancer. Breast J 13:274-280.

25. von Minckwitz G, Kümmel S, Vogel P et al (2008) German Breast Group.Intensified neoadjuvant chemotherapy in early-responding breast cancer: phase III randomized GeparTrio study. J Natl Cancer Inst 100:552-562.

26. Kong H, Huang ZH, Li Q, Yang LC, Yu JL, Li Z (2008) Adenovirus-mediated double suicide gene selectively kills breast cancer MCF-7 cells in vitro. Nan Fang Yi Ke Da Xue Xue Bao 28:907-910.

27. Yang WS, Park SO, Yoon AR, Yoo JY, Kim MK, Yun CO, Kim CW (2006) Suicide cancer gene therapy using pore-forming toxin, streptolysin O. Mol Cancer Ther 5:16101619. 
28. Brandtner EM, Kodajova P, Hlavaty J et al (2008) Reconstituting retroviral (ReCon) vectors facilitating delivery of cytotoxic genes in cancer gene therapy approaches. $\mathrm{J}$ Gene Med 10:113-122.

29. Janát-Amsbury MM, Yockman JW, Anderson ML, Kieback DG, Kim SW (2006) Combination of local, non- viral IL12 gene therapy and systemic paclitaxel chemotherapy in a syngeneic ID8 mouse model for human ovarian cancer. Anticancer Res 26:3223-3228.

30. Deharvengt S, Rejiba S, Wack S, Aprahamian M, Hajri A (2007) Efficient electrogene therapy for pancreatic adenocarcinoma treatment using the bacterial purine nucleoside phosphorylase suicide gene with fludarabine. Int J Oncol 30:1397-1406.

31. Abaza MS, Al-Saffar A, Al-Sawan S, Al-Attiyah R (2008) c-myc antisense oligonucleotides sensitize human colorectal cancer cells to chemotherapeutic drugs. Tumour Biol 29:287-303.

32. Fandy TE, Shankar S, Srivastava RK (2008) Smac/DIABLO enhances the therapeutic potential of chemotherapeutic drugs and irradiation, and sensitizes TRAIL-resistant breast cancer cells. Mol Cancer. doi:10.1186/1476-4598-7-60

33. Lu H, Chen CS, Waxman DJ (2009) Potentiation of methoxymorpholinyl doxorubicin antitumor activity by P450 3A4 gene transfer. Cancer Gene Ther 16:393-404.

34. Aroui S, Brahim S, De Waard M, Bréard J, Kenani A (2009) Efficient induction of apoptosis by doxorubicin coupled to cell-penetrating peptides compared to unconjugated doxorubicin in the human breast cancer cell line MDA-MB 231. Cancer Lett. doi:10.1016/ j.canlet.2009.04.044

35. Yuan Z, Yan F, Wang YS et al (2009) PNAS-4, a novel pro-apoptotic gene, can potentiate antineoplastic effects of cisplatin Cancer Chemother Pharmacol. doi: 10.1007/s00280-0090998-5 
36. McGrogan BT, Gilmartin B, Carney DN, McCann A (2008) Taxanes, microtubules and chemoresistant breast cancer. Biochim Biophys Acta 2008 1785:96-132.

37. Boyd M, Mairs SC, Stevenson K, Livingstone A, Clark AM, Ross SC, Mairs RJ (2002) Transfectant mosaic spheroids: a new model for evaluation of tumour cell killing in targeted radiotherapy and experimental gene therapy. J Gene Med 4:567-576.

38. Kostarelos K, Emfietzoglou D, Papakostas A, Yang WH, Ballangrud A, Sgouros G (2004) Binding and interstitial penetration of liposomes within avascular tumor spheroids. Int $\mathbf{J}$ Cancer 112:713-721.

39. Oktem G, Vatansever S, Ayla S, Uysal A, Aktas S, Karabulut B, Bilir A (2006) Effect of apoptosis and response of extracellular matrix proteins after chemotherapy application on human breast cancer cell spheroids. Oncol Rep 15:335-340.

40. Finocchiaro LM, Bumaschny VF, Karara AL, Fiszman GL, Casais CC, Glikin GC (2004) Herpes simplex virus thymidine kinase/ganciclovir system in multicellular tumor spheroids. Cancer Gene Ther 11:333-345.

41. Brandtner EM, Kodajova P, Hlavaty J et al (2008) Reconstituting retroviral (ReCon) vectors facilitating delivery of cytotoxic gene in cancer gene therapy approaches. J Gene Med 10:113-122. 
A

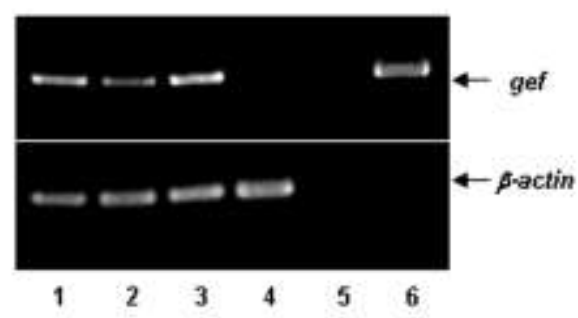

B

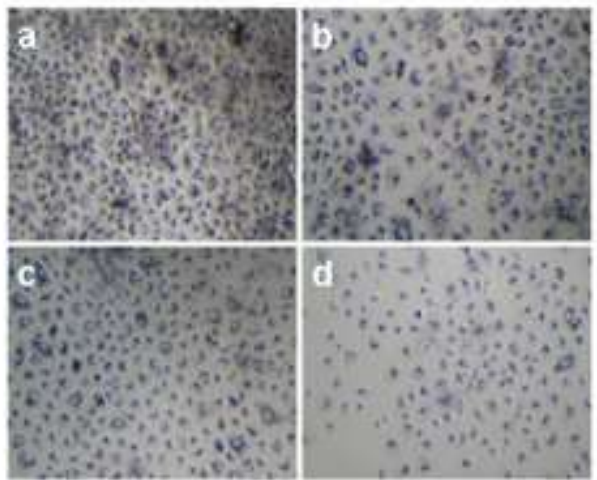

C
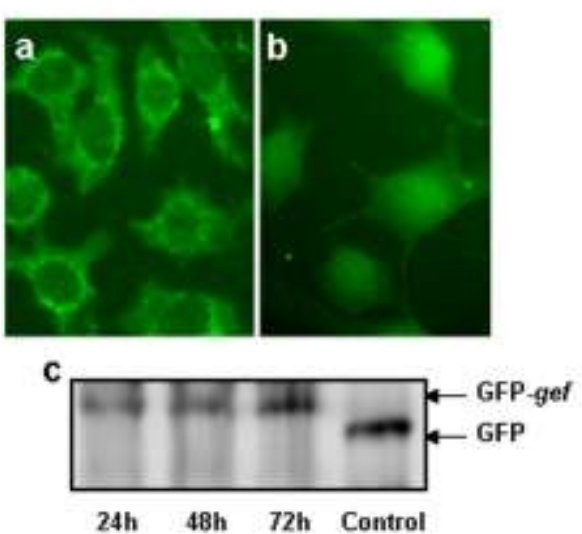
A
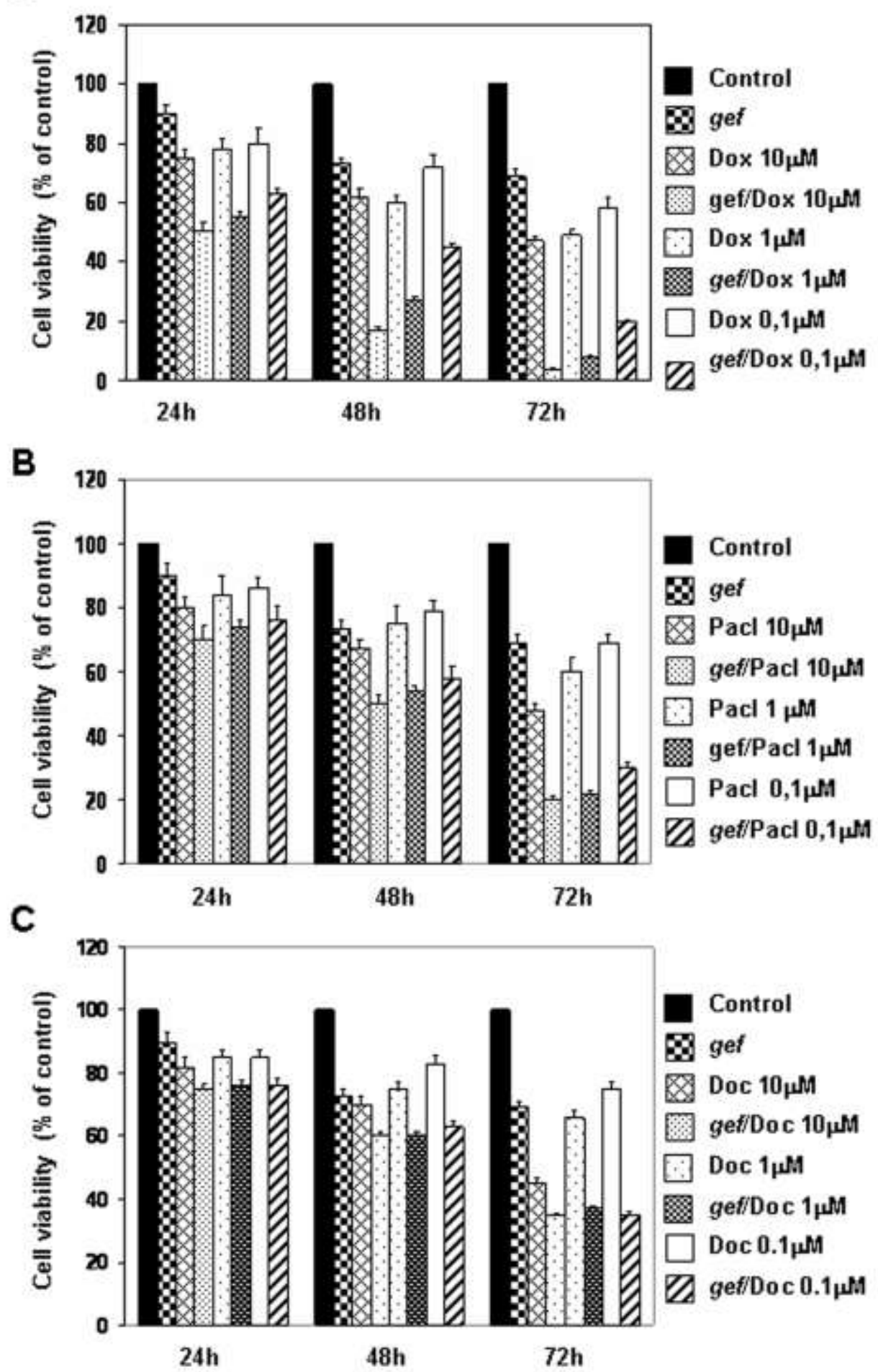
A
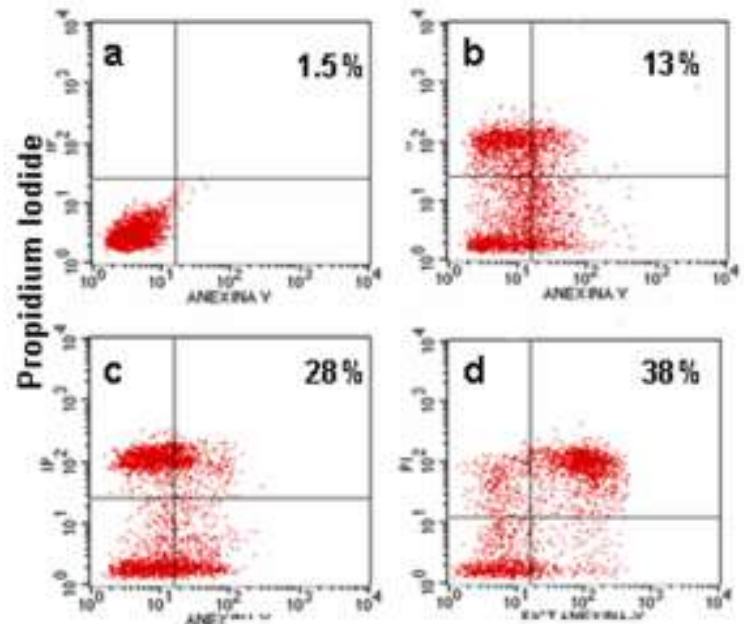

Annexin V.FITC

C
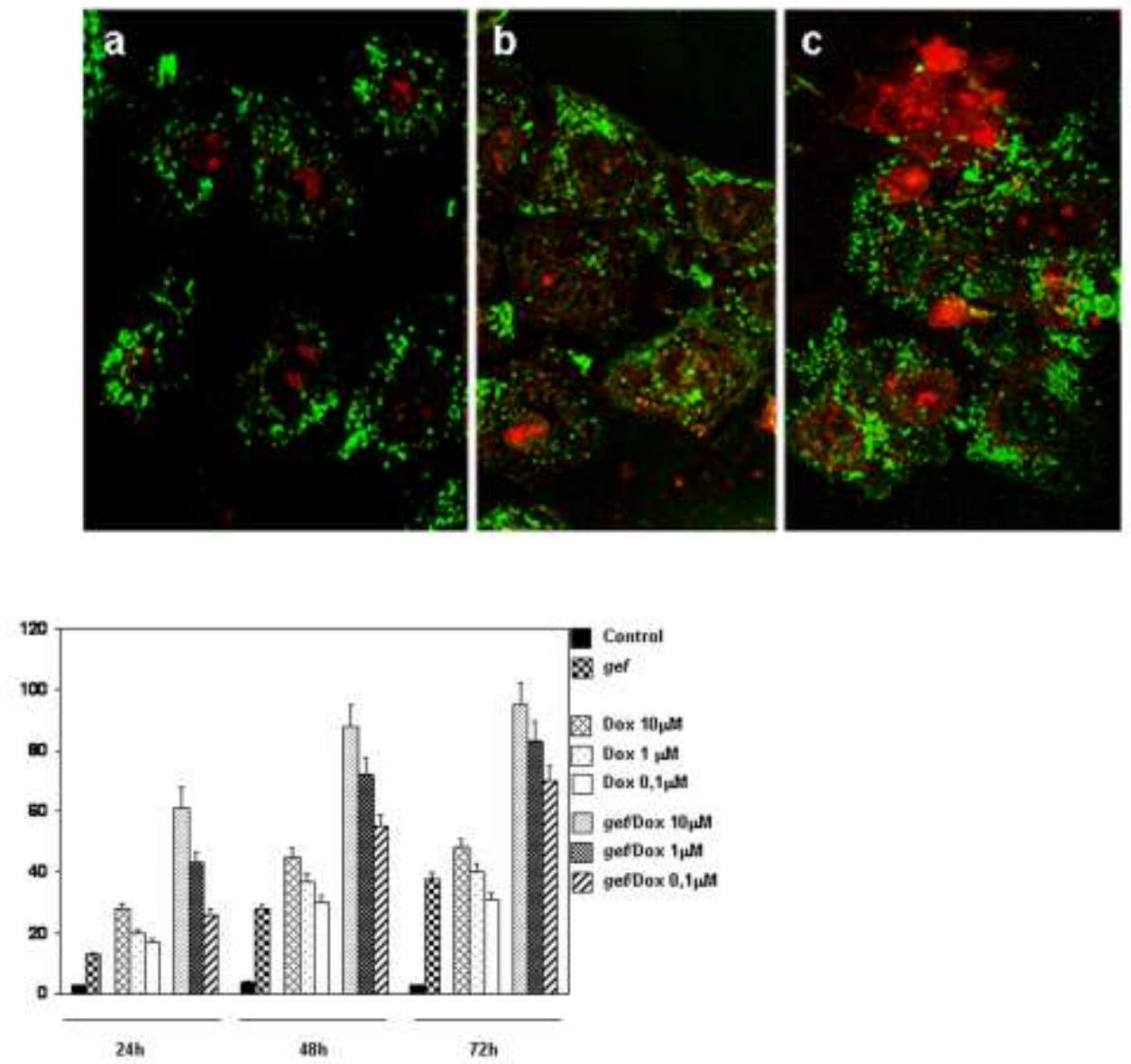

B

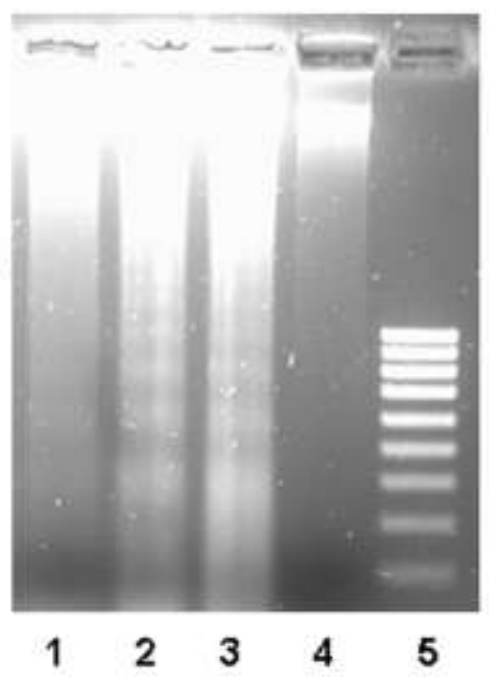

$\begin{array}{lllll}1 & 2 & 3 & 4 & 5\end{array}$ . 
A

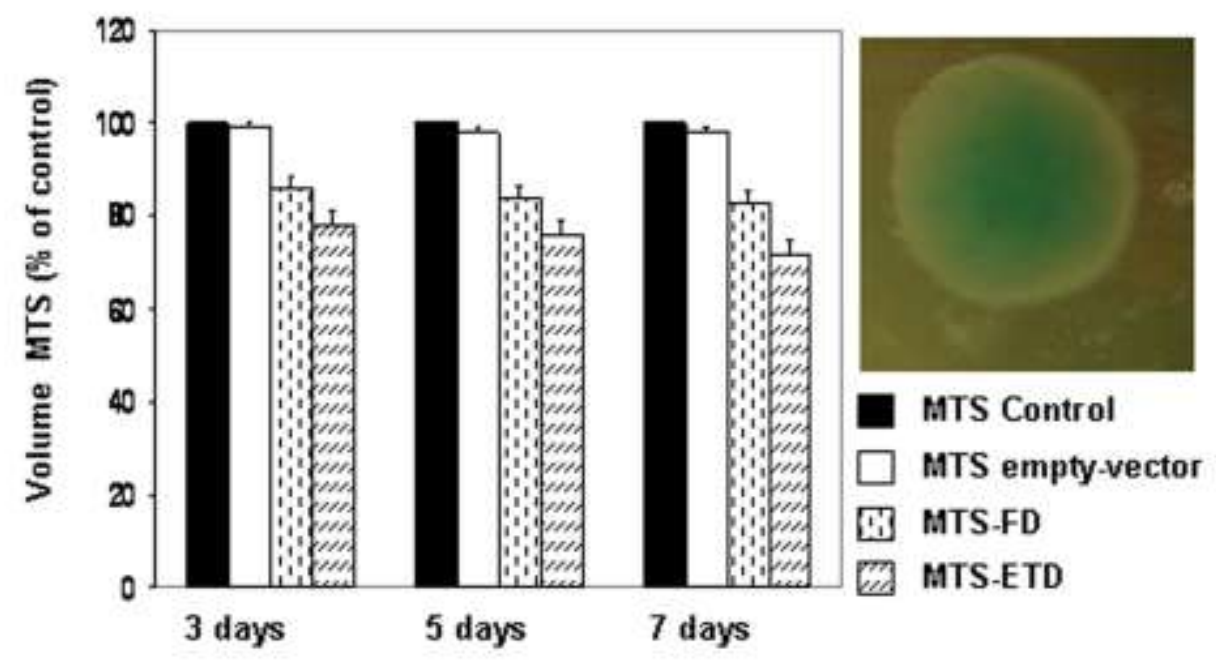

C
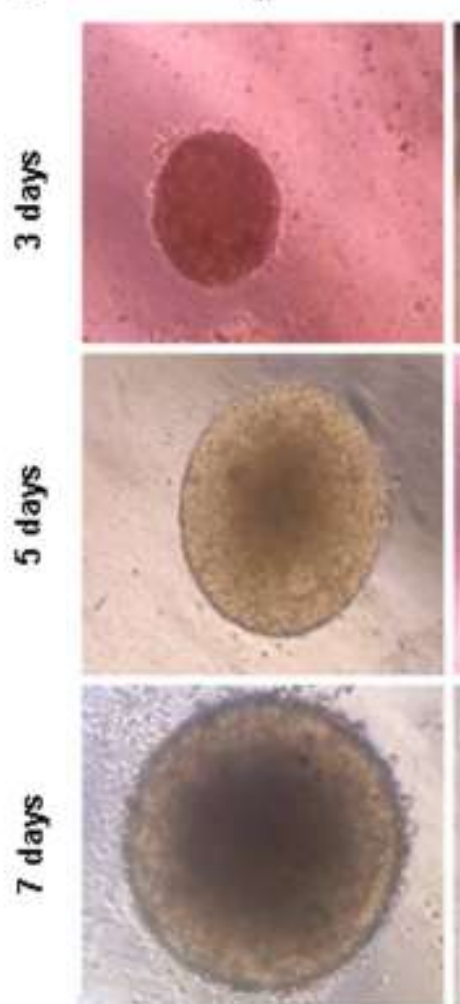

B

b
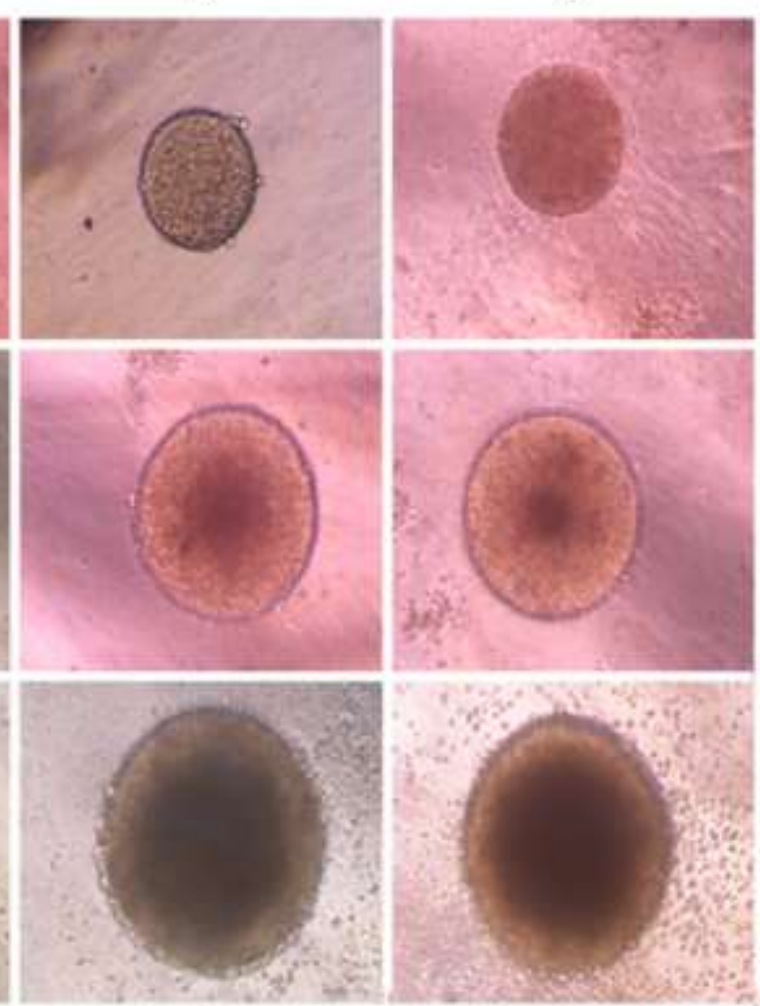
A a
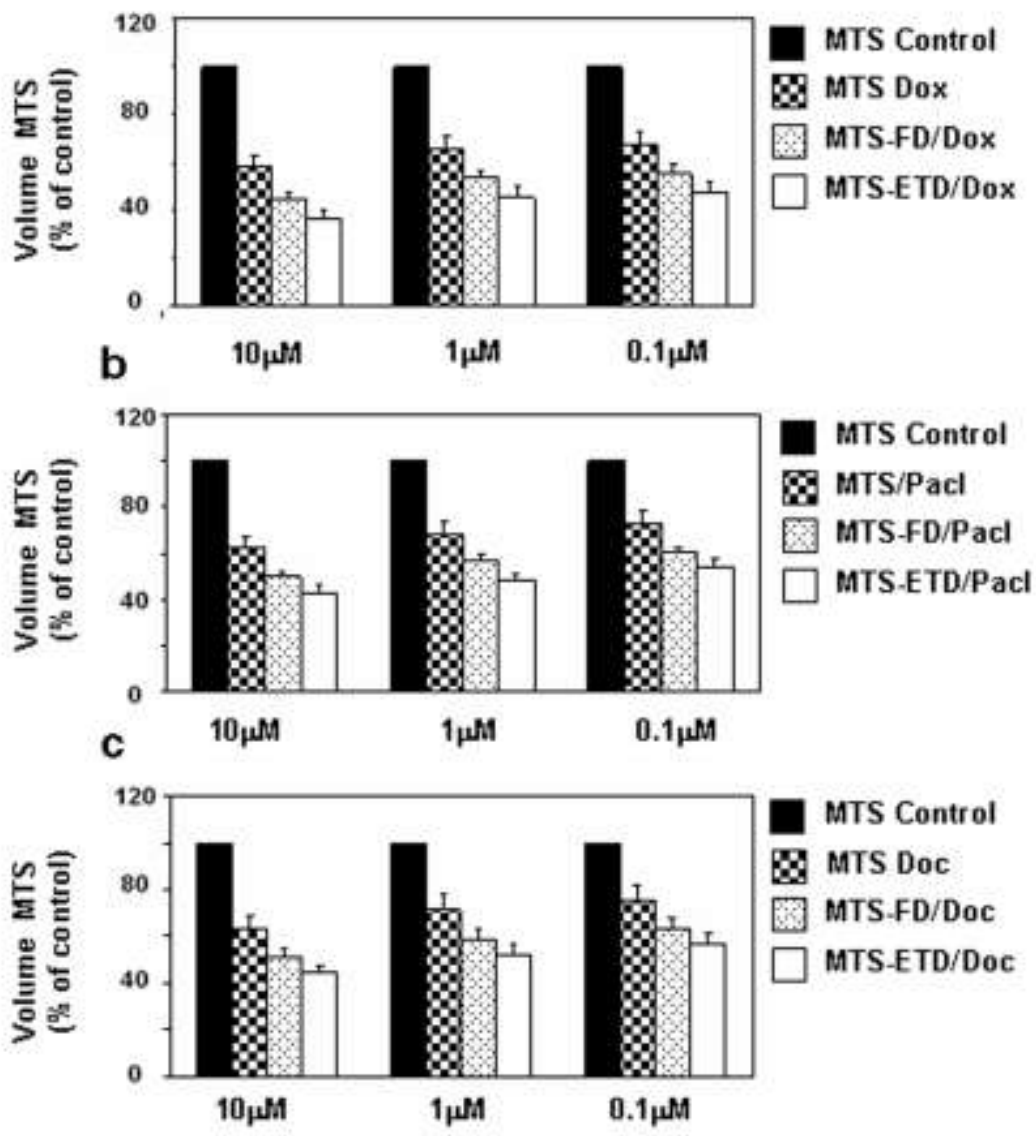

B a b

c d

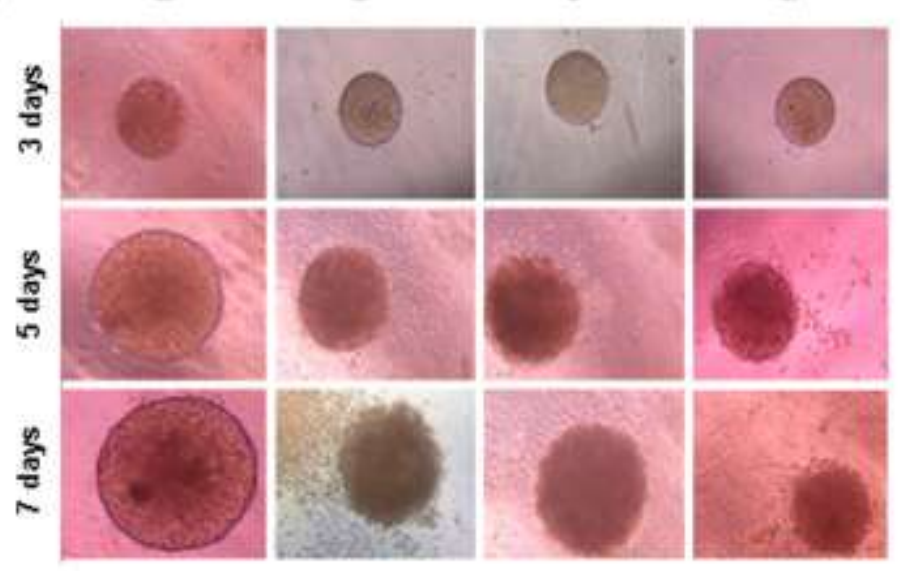

